\title{
Prof. Dr. Carl Kopp †
}

Am 24. Nov. 1912 erlag der a. o. Professor der Derniatologie an der Universität Münehen, Dr. med. Carl Kopp seinem sehweren, jalirelangen Leiden (Hodgkinsche Krankheit).

Kopp war der ßegründer einer Lehrstätte und einer Poliklinik für Haut- und Geschlechtskrankheiten. Nach langjähriger Assistententätigkeit bei Merkel, Ziemßen und Ríedinger wurde er von Neíßer für die Derniatologie gewonnen und ist einer seiner bekanntesten Schüler geworden. Im Jahre 1886 habilitierte er sich in Münehen, wo er weiterhin eine bedeutende öffentliehe wie private Tätigkeit entfaltete. 1899 erhielt er den Professortitel; 1910 wurde er etatsmäßig, nachdem er über 20 Jahre ohne jedes Entgelt und unter den unangenehmsten, gedrücktesten Verhältnissen seine Dienste unermüdlich der Poliklinik gewidmet hatte.

Trotz dieser mißlichen Arbeitsgelegenheiten hat er eine stattliche Reihe von Arbeiten (ca. 60) veröffentlicht, von denen ich hier nur die be-deutendsten erwähnen will: Die Trophoneurosen der Haut, Lehrbuch der venerisehen Erkrankungen, Atlas der Geschlechtskrankheiten, Atlas der Hautkrankheiten. Er war Mitarbeiter am Handbuch der speziellen Therapie innerer Krankheiten von Penzoldt und Stintzing und der Bibliothek der med. Wissenschaften von Dracke. Die Frage des Lupus erythematodes und der Leukoplakia mucosae fanden wiederholte Bearbeitung. Die Wasser-wann-Serodiagnose beurteilte er skeptisch, für die Salvarsanbehandlung trat ergleichein. Viel schrieb erüber dieBekämpfung der Geschlechtskrankheiten, die Prostitutionsfrage und das Gesehlechtliche in der Jugenderziehung.

Dieses ganze Gebiet interessiert $\theta$ ihn besonders, weil er 1903 einen Zweig der Deutschen Gesellschaft zur Bekämpfung der Geschlechtskrankheiten in Münehen gründete, als dessen Vorstand er dann viele Vorträge hielt.

In den letzten Jahren lag ihm aber besonders die Schaffung und Ausstattung der neuen, modern eingerichteten Poliklinik am Herzen. Leider sollte er die Früchte dieser großen Arbeit nicht recht genießen; er hat kaum noch Vorlesungen im neuen Hause halten können. Die Stimme versagte, der Tod war für ihn eine Erlösung. Er ist nur 57 Jahre alt geworden. Die Universität verliert in ihm einen ihrer beliebtesten Lehrer, der durch seine Beredsamkeit und sein diagnostisches Talent seine zahlreichen Schüler begeisterte. H. Ploeger-München.

Tagesnachriehten.

Ein neues Regkment für die ärztlichen Prüfungen ist kürzlich in der Schweiz ausgegeben worden. In diesem ist nun auch die Derniatologie als offizieiles Prüfungsfach aufgenommen worden, und zwar wird von den Studierenden der Medizin verlangt, daß sie, nachdem sie den Nach-weis der genügenden Ausbildung erbracht, ein Semester in einer dermato-logischen Klinik praktiziert haben. Sie werden dann im praktischen Teile des Staatsexamens geprüft, und zwar so, daß diese Prüfung mit eine Fachnote berechnet wird. Die Prüfung ist im wesentlichen eine praktische. Auf diese Weise ist die Derniatologie ebenso wie die Pädiatrie der Ophthalmologie bei den schweizerischen Prüfungen vollständig gleichgestellt. Hoffentlichwird nun 
auchDeutschlaiid bald folgenund der Derniatologie die Stellung geben, die sie in fast alien Nachbarländern bereits inne hat. 